\title{
Scintigraphic Imaging of Endothelium-Dependent Vasodilation in the Forearm A Preliminary Report
}

\author{
Alper O. Karacalioglu, MD; Sait Demirkol, MD*; Ozdes Emer, MD; Turgay Celik, MD*; \\ Selim Kilic, MD**; Seyfettin Ilgan, MD; Mehmet A. Ozguven, MD
}

\begin{abstract}
Background The diagnosis of endothelial dysfunction has been gaining clinical importance, but although endothelial function testing is available in the research setting, no technique yet exists that is simple, safe, reproducible and easily performed as a clinical screening method. The aim of this study was to design a new, scintigraphic method of imaging the flow-mediated dilation in the forearm, which represents the functional characteristic of endothelial dysfunction.

Methods and Results The study group comprised 118 subjects in whom left forearm ischemia was induced by inflating a sphygmomanometer cuff to supra systolic pressure for $4.5 \mathrm{~min}$. Later, dynamic acquisition $(2 \mathrm{~s}$ frame/min) was initiated after the injection of technetium-99m methoxy-isobutyl isonitril into the dorsal pedal veins. Equivalent regions of interest were drawn on both arms to detect total activity counts during $1 \mathrm{~min}$ and the perfusion ratios (left arm/right arm) were calculated. The left arm counts $(22,203.3 \pm 12,372.7)$ were significantly higher than the right arm counts $(9,980.9 \pm 5,931.9)(\mathrm{p}<0.001)$. A significant decrease in perfusion ratios was noted in the hypertension and hypercholesterolemia groups. An increase in the number of risk factors caused an insignificant decrease in perfusion ratio $(\mathrm{p}=0.346)$.

Conclusion Non-invasive evaluation of endothelium-dependent vasodilation by semiquantitative scintigraphic method using radioactive perfusion tracer provided promising results. (Circ J 2006; 70: 311-315)
\end{abstract}

Key Words: Endothelial dysfunction; Flow-mediated vasodilation; Nitrous oxide; Scintigraphy; SPECT; 99mTc-MIBI

$\mathbf{T}$ he endothelium is the monolayer of the endothelial cells lining the lumen of all blood vessels and functions as a large autocrine, paracrine, and endocrine organ that regulates vasoactivity, smooth-muscle-cell proliferation, platelet aggregation, monocyte adhesion, hemostasis, thrombolysis, inflammation, immune responses, and free radical production!,2 The term "endothelial dysfunction" is most often used to denote impairment of endothelium-dependent vasodilation, but probably encompasses those conditions leading to endothelial activation with abnormalities in endothelial interactions with leukocytes, platelets and regulatory substances? Recent insights into the basic mechanisms involved in atherogenesis indicate that deleterious alterations of endothelial physiology represent a key early step in the development of atherosclerosis and are also involved in plaque progression and the occurrence of atherosclerotic complications4-6 Several studies have shown that risk factor modification improves endothelial function, and therefore, the diagnosis of this clinical entity has been gaining importance?-11

Because it is a diffuse disease process, endothelial function can be assessed clinically either invasively or nonin-

(Received August 5, 2005; revised manuscript received November 24, 2005; accepted December 6, 2005)

Departments of Nuclear Medicine, *Cardiology and **Public Health and Epidemiology, Gulhane Military Medical Academy and School of Medicine, Etlik-Ankara, Turkey

Mailing address: Alper O. Karacalioglu, MD, Gulhane Military Medical Academy and School of Medicine, Department of Nuclear Medicine, 06018 Etlik-Ankara, Turkey. E-mail: aokaracali@yahoo.com vasively in the coronary artery and peripheral circulation by monitoring the vasodilatation produced by the administration of endothelium-dependent agonists or by increased blood-flow shear (flow-mediated vasodilation)!2-16 Although endothelial function testing is available in the research setting, no technique yet exists that is simple, safe, reproducible and easily performed as a screening method. As a simple noninvasive test for endothelial dysfunction would potentially have immediate clinical applicability, there is growing interest in developing a noninvasive, reliable method of assessment. Such a test might identify presymptomatic subjects at high risk of atherosclerotic complications and therefore allow specific "targeting" of primary preventive strategies.

The aim of this prospective study was to design a new, semiquantitative scintigraphic method of imaging the flowmediated vasodilation in the forearm, which represents the functional characteristic of the endothelial dysfunction.

\section{Methods}

\section{Patient Population}

This study was designed to be part of the myocardial perfusion single photon emission computed tomography (SPECT) imaging (MPI) in order to decrease the patients' radiation burden and to obviate another imaging session. We studied 118 outpatients (72 men, 46 women; age range, 20-80 years; mean age $\pm S D, 45.2 \pm 12.4$ ) referred for MPI because of risk factors for coronary artery disease. They did not have known complicated cardiovascular disease. 


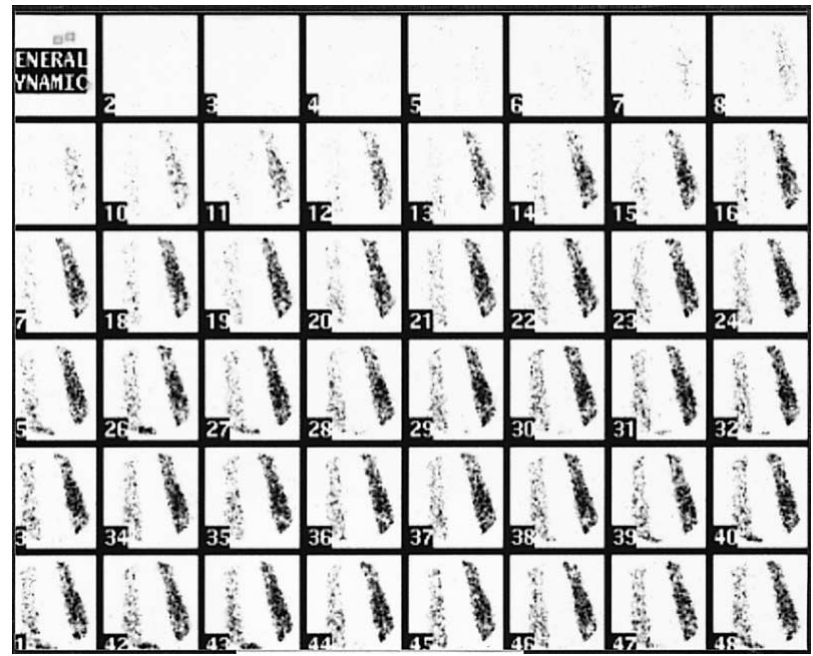

Fig 1. Sequential 1-s dynamic images after release of the tourniquet and IV injection of $20 \mathrm{mCi}$ technetium-99m methoxy-isobutyl isonitril demonstrate reactive hyperemia or flow-mediated vasodilatation in the left arm of a patient.

A structured interview was performed and clinical history acquired, and cardiac risk factors were assessed before nuclear testing. Patients were divided into subgroups according to their risk factors described as follows. Hypertension was defined as repeated blood pressure measurements $>140 / 90 \mathrm{mmHg}$ or antihypertensive medication (17 patients were on angiotensin converting enzyme inhibitor treatment, 9 patients were on calcium-channel blocker treatment and 7 patients were on $\beta$-blocker treatment). Diabetes mellitus was defined as a fasting glucose level $\geq 7.8 \mathrm{mmol} / \mathrm{L}$ or the need for insulin or oral hypoglycemic agents. Hypercholesterolemia was defined as a total cholesterol level $\geq 6.4 \mathrm{mmol} / \mathrm{L}$ or treatment with lipid-lowering medication (10 patients were on statin treatment). The diagnosis of coronary artery disease was based on the standard criteria of MPI, electrocardiographic changes, and clinical history. The study population was also divided into 2 subgroups according to the scintigraphic findings reported by 2 expe- rienced observers in a blinded manner: patients in group 1 had normal perfusion patterns, and patients in group 2 had perfusion defects on their scans.

Patients were fasted for at least $6 \mathrm{~h}$ before the study, and they were studied in a quiet, temperature-controlled room. All vasoactive medications were withheld for at least $24 \mathrm{~h}$. Patients with non-satisfactory injection into their dorsal pedal veins and known bilateral lower extremity venous disease were excluded. All patients were informed about the procedure and verbal informed consent was given before the test. The hospital ethics committee approved the study protocol.

\section{Technique, Acquisition Protocol and Instrumentation}

All patients underwent 2-day (stress-rest technetium-

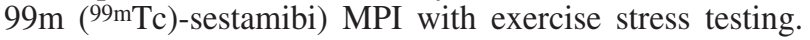
Following symptom-limited treadmill exercise test using the standard Bruce's protocol, 740-925 MBq (20-25 mCi) of 99mTc-sestamibi (reconstituted from Cardio-SPECT kits, Medi-Radiopharma, Budapest, Hungary) was injected intravenously at peak stress. Stress SPECT imaging was initiated 30-60 min later, using a $15 \%$ window centered over the $140-\mathrm{keV}$ photopeak. Acquisitions were performed using a 2-detector $90^{\circ}$ camera (Optima; GE, Milwaukee, WI, USA) with 64 projections over $180^{\circ}$ (right anterior oblique $45^{\circ}$ to left posterior oblique $45^{\circ}$ ).

Next day, while the patient remained seated both forearms were placed on the gamma camera (Starcam; GE, Milwaukee, WI, USA), equipped with low energy generalpurpose collimator. A sphygmomanometer cuff was placed around the left arm just above the elbow and inflated to supra systolic pressure for $4.5 \mathrm{~min}$ to induce forearm ischemia by interrupting arterial blood supply. Then 740 $925 \mathrm{MBq}(20-25 \mathrm{mCi}){ }^{99 \mathrm{~m} T c}$ methoxy-isobutyl isonitrile (MIBI) prepared for rest MPI was injected into the dorsal pedal veins of the patient. When activity was detected in the right arm on the monitor, the cuff was deflated and dynamic acquisition ( $2 \mathrm{~s}$ per frame/min) was initiated simultaneously (Fig 1). Rest SPECT imaging was carried out $45-60$ min later in all cases.
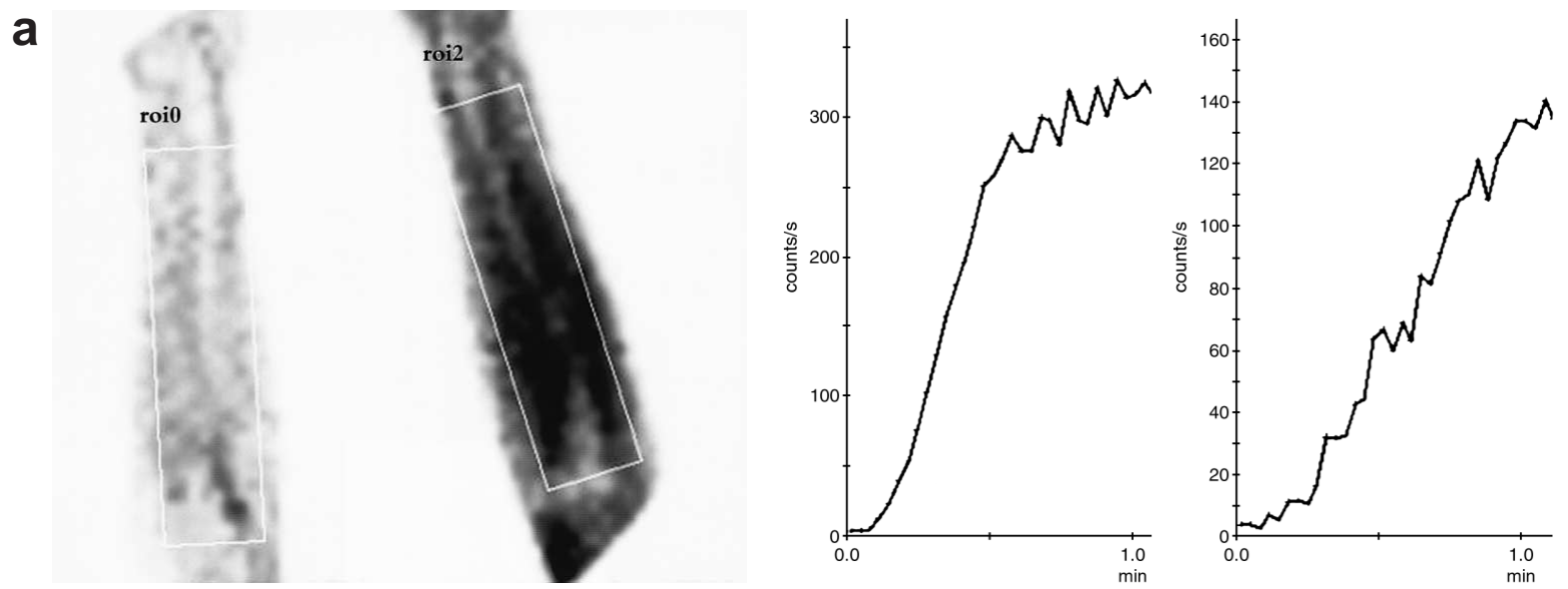

Fig 2. Regions of interest drawn in approximately similar locations on the reframed image (a). Increased perfusion in the left arm of the patient can be easily seen. The left time-activity curve demonstrates dual phase; the early rapid and late slow activity increase signifies flow-mediated vasodilation in the left arm of the patient (b), whereas the one on the right shows approximately proportional activity increase, signifying normal perfusion pattern in the right arm of the same patient. 
Table 1 Scintigraphic Results

\begin{tabular}{|c|c|c|c|c|c|}
\hline & \multicolumn{5}{|c|}{ Perfusion ratios* } \\
\hline & $n(\%)$ & Positive & $n(\%)$ & Negative & p value $* *$ \\
\hline \multicolumn{6}{|l|}{ Risk factors } \\
\hline Diabetes mellitus & $13(11)$ & $2.20 \pm 0.52$ & $105(89)$ & $2.36 \pm 0.59$ & 0.355 \\
\hline Hypertension & $33(28)$ & $2.05 \pm 0.50$ & $85(72)$ & $2.45 \pm 0.57$ & 0.001 \\
\hline Family history & $18(15)$ & $2.41 \pm 0.50$ & $100(85)$ & $2.33 \pm 0.59$ & 0.619 \\
\hline Hypercholesterolemia & $13(11)$ & $2.04 \pm 0.47$ & $105(89)$ & $2.38 \pm 0.58$ & 0.044 \\
\hline Smoking & $70(59)$ & $2.36 \pm 0.58$ & $48(41)$ & $2.31 \pm 0.58$ & 0.575 \\
\hline \multicolumn{6}{|l|}{ Gender } \\
\hline$M$ & $72(61)$ & $2.35 \pm 0.58$ & & & 0.818 \\
\hline F & $46(39)$ & $2.33 \pm 0.59$ & & & 0.818 \\
\hline \multicolumn{6}{|l|}{ Age (years) } \\
\hline$\geq 60$ & $23(19)$ & $2.10 \pm 0.56$ & & & \\
\hline $50-59$ & $30(25)$ & $2.43 \pm 0.56$ & & & \\
\hline $40-49$ & $29(24)$ & $2.24 \pm 0.51$ & & & \\
\hline$\leq 39$ & $36(30)$ & $2.52 \pm 0.57$ & & & \\
\hline
\end{tabular}

$*$ Mean $\pm S D, * * i n d e p e n d e n t$ samples $t$-test.

\section{Image Evaluation}

The left and right arms of the patient were used as test and control groups respectively to detect reactive hyperemia in this study. Dynamic images were reframed as 1 image and equivalent regions of interest (ROIs) were drawn in approximately similar locations on both arms in order to detect total activity counts during $1 \mathrm{~min}$ (Fig 2a). Counts derived from the left forearm ROI were divided by the counts derived from the right forearm ROI to calculate the left arm/right arm perfusion ratio, showing the response to shear stress of the test arm for comparative studies. Later, time-activity curves were derived and injection quality was assessed by observing the slopes and time-to-peak times (Fig 2b).

\section{Statistical Analyses}

Because many known and unknown factors affect endothelial functions, we could not identify a normal group. The counts derived from ROIs are presented as counts per min (cpm) and the perfusion ratios of the risk groups are presented as the mean \pm SD. The difference between counts derived from the ROIs of the left and right arms and the difference between the perfusion ratios of patients with or without perfusion defects on their scans were tested for significance by paired samples t-test. The difference between the patients with or without same risk factor was tested for significance by independent samples t-test, and the difference among groups of increasing risk factors was tested for significance by one-way ANOVA test. Statistical analysis was performed with the SPSS 10.0 Statistical Package Program for Windows (SPSS Inc, Chicago, IL, USA). Differences were considered significant at $\mathrm{p}<0.05$.

\section{Results}

The means and standard deviations of the counts derived from the ROIs of the left and right arms of the patients were $22,203.3 \pm 12,372.7$ and 9,980.9 $\pm 5,931$ respectively. The left arm counts signifying increased perfusion secondary to flow-mediated vasodilation were significantly higher than those of the right arms, which signify the baseline perfusion $(\mathrm{p}<0.001)$.

The perfusion ratios varied from 1.43 to 3.10 . A significant decrease in perfusion ratio was noted in the hypertension and hypercholesterolemia groups (Table 1).

The effect of an increase in the number of risk factors on
Table 2 Cumulative Effects of the Risk Groups

\begin{tabular}{ccccc}
\hline \hline Group & $\begin{array}{c}\text { Riskfactors } \\
(n)\end{array}$ & $\begin{array}{c}\text { Patients } \\
(n)\end{array}$ & $\begin{array}{c}\text { Perfusion } \\
\text { ratios* }\end{array}$ & p value** \\
\hline 1 & 1 & 37 & $2.53 \pm 0.57$ & \\
2 & 2 & 32 & $2.44 \pm 0.59$ & \\
3 & 3 & 23 & $2.24 \pm 0.52$ & 0.346 \\
4 & 4 & 19 & $2.11 \pm 0.51$ & \\
5 & 5 & 7 & $2.10 \pm 0.67$ & \\
\hline
\end{tabular}

*Mean $\pm S D, * *$ one-way ANOVA test.

the perfusion ratio is shown in Table2. Although an increase in the number of risk factors caused a decrease in perfusion ratios generally, it was not statistically significant ( $\mathrm{p}=0.346)$.

According to the MPI results, 41 (35\%) of 118 patients had perfusion defects on their scans. There was not a statistically significant difference between the perfusion ratios of the patients with $(2.37 \pm 0.64)$ and without $(2.32 \pm 0.53)$ perfusion defects $(\mathrm{p}=0.66)$.

Dynamic, reframed images and time-activity curves derived from the ROIs of a representative case are shown in Figs 1 and 2. The left arm curve was usually biphasic and first steep increase was usually followed by a plateau or slight increase (Fig $2 b$ ). A proportional increase was usually detected in the time-activity curve of the right arm.

\section{Discussion}

The local release of nitric oxide (NO) in response to shear stress and hypoxia causes reactive hyperemia by dilating the distal microvasculature. In our study using a perfusion tracer, we detected a significant increase in the perfusion of the left arm of the patients after an ischemic period and therefore, we consider that our scintigraphic method is highly effective in demonstrating the vascular dilator response to shear stress. Injection of $99 \mathrm{mTc}$-MIBI into dorsal pedal veins of the patient theoretically provides standardization in the amount of radioactivity reaching both brachial arteries by eliminating differences in the amount of injected activity and injection volume. Because activities were counted from ROIs of the same size drawn on both forearms under the same conditions, quantitative data derived from this scintigraphic method are comparable. Furthermore, the calculated perfusion ratios indicating 
the endothelium-dependent vasodilation capacity of the test arm of the patient enables comparison of risk groups.

Endothelial function is impaired by all the known coronary risk factors, including advanced age, male gender, dyslipidemia, hypertension, smoking and diabetes mellitus, as well as other unknown risk factors ${ }^{17,18}$ After the evaluation of the risk groups in our study, it was noted that the perfusion ratios were lower in the hypertension and hypercholesterolemia groups. We also expected to find lower perfusion ratios in the other risk groups, but in reality it is did not occur, because of individual differences in endothelial function, which has been pointed out by studies showing that individuals with normal endothelial function and patients with various stages of endothelial dysfunction do not necessarily differ in their risk factor profiles. ${ }^{19-21}$ Besides, the presence of established cardiovascular risk factors is not the only determinant of endothelial function. Rather, endothelial integrity depends on the balance of all cardiovascular risk factors and vasculoprotective elements in a given individual, including as yet unknown variables and genetic predisposition.22 This may also explain the absence of a significant difference between the perfusion ratios of patients with and without perfusion defects on their scans. On the other hand, some studies have indicated that the risk of developing endothelial dysfunction increases with the number of risk factors present in an individual23,24 Our findings are also consistent with those results.

The brachial artery dilator response to shear stress has been shown to be reproducible, ${ }^{12}$ caused mainly by endothelial release of $\mathrm{NO}, 25,26$ and to correlate well with the results of invasive testing of coronary endothelial function. ${ }^{27}$ Current methods of assessing endothelial function by measuring brachial artery flow-mediated vasodilatation vary significantly in terms of cost, invasiveness and standardization of the technique, ${ }^{12,13,18,28}$ but our method is minimally invasive, relatively easy to perform and gives objective quantitative results. It has also the potential to be easily incorporated into any center with nuclear medicine facilities.

Our study differs in a few points from a similar-aimed study 29 First, we used the dorsal pedal veins instead of the brachial veins and for this reason we did not need to use a catheter for injection. Second, although we released the cuff as soon as we detected activity in the control arm in order to detect the actual effect of NO, there was a lag time of $30 \mathrm{~s}$, which is important because the biologic half-life of NO is approximately $5 \mathrm{~s}^{30,31}$ and flow-mediated vasodilatation is mediated mainly by NO. Third, Dupuis et al used a dynamic imaging time of $1 \mathrm{~s}$ frame for $10 \mathrm{~min}$ and $99 \mathrm{mTc}$ tetrofosmin as the perfusion tracer. Probably for these reasons our time-activity curves were different and we did not detect the peak time points. Finally, they described a control group with a negative symptom-limited exercise stress test, and patients in that group had 1 known traditional risk factor for coronary artery disease, apart from older age, and they did not undergo stress myocardial perfusion imaging. However, endothelial function can be affected by many known and unknown risk factors and therefore we could not describe a control group. Probably for these reasons, our results are different and we could not find a significant difference between the results of patients with and without detectable coronary artery disease.

\section{Study Limitations}

First, intravenous injection of the radiopharmaceutical into the dorsal pedal veins was sometimes difficult but may become easy in time in the hands of an experienced operator. Second, numerous factors affect flow-mediated vascular reactivity, including temperature, food, drugs and sympathetic stimuli, among others and these factors are important for the reproducibility of this method and it may not always be possible to take all these confounding factors into considering when preparing a subject for the study. Third, because it was part of the myocardial perfusion SPECT study, we used 99mTc-MIBI as a radiopharmaceutical, but other perfusion tracers, such as $99 \mathrm{~m}$ Tc-diethylenetriamine pentaacetic acid, may give better results regarding the blood flow in the conduit and resistant vessels of the arm without muscular uptake in a separate study. Fourth, our study population was relatively young and therefore the incidences of major risk were infrequent. Finally, because the right arm of the patient was the control group, this gave us an opportunity to overcome differences in injection sites, bolus amount of activity, injection volume, route of the radiopharmaceutical and arrival of the tracer to the arms. At the same time, this approach may be the cause of error because being right- or left-handed may change the baseline blood flows in both arms.

The impaired endothelial function that occurs in atherosclerosis and in the presence of risk factors for atherosclerosis plays an important role in the subsequent development of acute coronary syndromes. It is also important in microvascular ischemia syndromes, such as 'Syndrome $\mathrm{X}$ ' which is characteristically found in individuals with chest pain but no hemodynamically significant coronary artery stenosis 32 Therefore, evaluation of endothelial function in these patients may be helpful in differential diagnosis. Although our method is an initial step and further characterization is required, we believe that we have opened a window to a new research area.

\section{Conclusion}

We imaged the flow-mediated-dilation in the forearm, which represents the functional characteristic of endothelial function, with a semiquantitative scintigraphic method using perfusion tracer and obtained quantitative data. Further correlative and comparative studies with other defined methods are necessary to establish its efficacy in clinical practice.

\section{References}

1. Moncada S, Higgs A. The L-arginine-NO pathway. $N$ Engl J Med 1993; 329: 2002-2012.

2. Meredith IT, Anderson TJ, Uehata A, Yeung AC, Selwyn AP, Ganz $\mathrm{P}$. Role of endothelium in ischemic coronary syndromes. Am J Cardiol 1993; 72: 27C-31C.

3. Bonetti PO, Lerman LO, Lerman A. Endothelial dysfunction: A marker of atherosclerotic risk. Arterioscler Thromb Vasc Biol 2003; 23: $168-175$.

4. Ross R. Atherosclerosis; an inflammatory disease. $N$ Engl J Med 1999; 340: 115-126.

5. Kinlay S, Ganz P. Role of endothelial dysfunction in coronary artery disease and implications for therapy. Am J Cardiol 1997; 80: 11-I16-I.

6. Cai H, Harrison DG. Endothelial dysfunction in cardiovascular diseases: The role of oxidant stress. Circ Res 2000; 87: 840-844.

7. Paterick TE, Fletcher GF. Endothelial function and cardiovascular prevention: Role of blood lipids, exercise, and other risk factors. Cardiol Rev 2001; 9: 282-286.

8. Suwannaprapha P, Chaisri U, Riyong D, Maneerat Y. Improvement of function and morphology of tumor necrosis factor-alpha treated endothelial cells with 17-beta estradiol: A preliminary study for a fea- 
sible simple model for atherosclerosis. Circ J 2005; 69: 730-738.

9. Ikeda Y, Biro S, Kamogawa Y, Yoshifuku S, Eto H, Orihara K, et al. Repeated thermal therapy upregulates arterial endothelial nitric oxide synthase expression in Syrian golden hamsters. Jpn Circ J 2001; 65: 434-438.

10. Morita H, Saito Y, Ohashi N, Yoshikawa M, Katoh M, Ashida T, et al. Fluvastatin ameliorates the hyperhomocysteinemia-induced endothelial dysfunction: The antioxidative properties of fluvastatin. Circ J 2005; 69: 475-480.

11. Takayama T, Wada A, Tsutamoto T, Ohnishi M, Fujii M, Isono T, et al. Contribution of vascular NAD $(\mathrm{P}) \mathrm{H}$ oxidase to endothelial dysfunction in heart failure and the therapeutic effects of HMG-CoA reductase inhibitor. Circ J 2004; 68: 1067-1075.

12. Sorensen KE, Celermajer DS, Spiegelhalter DJ, Georgakopoulos D, Robinson J, Thomas O, et al. Non-invasive measurement of human endothelium dependent arterial responses: Accuracy and reproducibility. Br Heart J 1995; 74: 247-253.

13. Corretti MC, Anderson TJ, Benjamin EJ, Celermajer D, Charbonneau F, Creager MA, et al. Guidelines for the ultrasound assessment of endothelial-dependent flow-mediated vasodilation of the brachial artery: A report of the International Brachial Artery Reactivity Task Force. J Am Coll Cardiol 2002; 39: 257-265.

14. Celermajer DS, Sorensen KE, Gooch VM, Spiegelhalter DJ, Miller OI, Sullivan ID, et al. Non-invasive detection of endothelial dysfunction in children and adults at risk of atherosclerosis. Lancet 1992; 340: $1111-1115$.

15. Higashi Y, Yoshizumi M. New methods to evaluate endothelial function: Method for assessing endothelial function in humans using a strain-gauge plethysmography: Nitric oxide-dependent and -independent vasodilation. J Pharmacol Sci 2003; 93: 399-404

16. Doucette JW, Corl PD, Payne HM, Flynn AE, Goto M, Nassi M, et al. Validation of a Doppler guide wire for intravascular measurement of coronary artery flow velocity. Circulation 1992; 85: 1899-1911.

17. Kuvin JT, Karas RH. Clinical utility of endothelial function testing: Ready for prime time? Circulation 2003; 107: 3243-3247.

18. Anderson TJ. Assessment and treatment of endothelial dysfunction in humans. J Am Coll Cardiol 1999; 34: 631-638.

19. Al Suwaidi J, Hamasaki S, Higano ST, Nishimura RA, Holmes DR Jr, Lerman A. Long-term follow-up of patients with mild coronary artery disease and endothelial dysfunction. Circulation 2000; 101: 948-954.

20. Halcox JPJ, Schenke WH, Zalos G, Mincemoyer R, Prasad A, Waclawiw MA, et al. Prognostic value of coronary vascular endothe- lial dysfunction. Circulation 2002; 106: 653-658.

21. Gokce N, Keaney JF Jr, Hunter LM, Watkins MT, Menzoian JO, Vita JA. Risk stratification for postoperative cardiovascular events via noninvasive assessment of endothelial function: A prospective study. Circulation 2002; 105: 1567-1572.

22. Gimbrone MA Jr, Topper JN, Nagel T, Anderson KR, GarciaCardeña G. Endothelial dysfunction, hemodynamic forces, and atherogenesis. Ann NY Acad Sci 2000; 902: 230-239.

23. Vita JA, Treasure CB, Nabel EG, McLenachan JM, Fish RD, Yeung $\mathrm{AC}$, et al. Coronary vasomotor response to acetylcholine relates to risk factors for coronary artery disease. Circulation 1990; 81: 491 497.

24. Celermajer DS, Sorensen KE, Bull C, Robinson J, Deanfield JE. Endothelium-dependent dilation in the systemic arteries of asymptomatic subjects relates to coronary risk factors and their interaction. J Am Coll Cardiol 1994; 24: 1468-1474.

25. Joannides R, Haefeli WE, Linder L, Richard V, Bakkali EH, Thuillez $\mathrm{C}$, et al. Nitric oxide is responsible for flow-dependent dilatation of human peripheral conduit arteries in vivo. Circulation 1995; 91: $1314-1319$.

26. Miura H, Wachtel RE, Liu Y, Loberiza FR Jr, Saito T, Miura M, et al. Flow-induced dilation of human coronary arterioles: Important role of $\mathrm{Ca}(2+)$-activated $\mathrm{K}(+)$ channels. Circulation 2001; 103: $1992-$ 1998.

27. Anderson TJ, Uehata A, Gerhard MD, Meredith IT, Knab S, Delagrange $\mathrm{D}$, et al. Close relation of endothelial function in the human coronary and peripheral circulations. J Am Coll Cardiol 1995; 26: $1235-1241$

28. Kuvin J, Patel A, Karas R. Need for standardization of non-invasive assessment of vascular endothelial function. Am Heart $J$ 2001; 141: $327-328$.

29. Dupuis J, Arsenault A, Meloche B, Harel F, Staniloae C, Gregoire J. Quantitative hyperemic reactivity in opposed limbs during myocardial perfusion imaging: A new marker of coronary artery disease. $J$ Am Coll Cardiol 2004; 44: 1473-1477.

30. Archer S. Measurement of nitric oxide in biological models. FASEB $J$ 1993; 7: 349-360.

31. Nathan C. Nitric oxide as a secretory product of mammalian cells. FASEB J 1992; 6: 3051-3064.

32. Pasqui AL, Puccetti L, Di Renzo M, Bruni F, Camarri A, Palazzuoli A, et al. Structural and functional abnormality of systemic microvessels in cardiac syndrome X. Nutr Metab Cardiovasc Dis 2005; 15: $56-64$. 